\title{
Research
}

Victoria S Hammersley, Jessica Harris, Aziz Sheikh, Emma Davidson and Samantha Walker

\section{Developing and testing of a screening tool to predict people without IgE-mediated allergy:}

\author{
a quantitative analysis of the predictive value of a screening tool
}

\begin{abstract}
Background

Consultations in primary care for allergies are common. It can be difficult to differentiate between IgE-mediated (atopic) symptoms which respond to allergen-specific interventions - and those that are non-atopic, without performing objective tests that are largely unavailable in UK general practice.

\section{Aim}

To develop and test a screening tool that can accurately discriminate between atopic and non-atopic individuals.
\end{abstract}

\section{Design and setting}

A validation study that took place in 2012 in adult volunteers aged $>16$ years in Scotland.

\section{Method}

A questionnaire screening tool was developed using questions from a large cohort study and through consultation with experts. Participants answered the questions and had skin prick tests for four aeroallergens (house dust mite, cat, dog, and mixed grasses). Participants were classified as atopic if any average wheal diameter was $\geq 3 \mathrm{~mm}$ bigger than the negative control. Sensitivity, specificity, and positive and negative predictive values of individual and combinations of questions were calculated.

\section{Results}

In all, 143 participants completed the questionnaire and underwent skin prick testing Of these, 81 (56.6\%) were atopic. Negative predictive values for the individual questions ranged from $48.2 \%$ (55 not atopic out of 114 negative answers) to $72.0 \%$ (18/25). An optimum combination of four questions was identified, in which a negative answer to all four questions was reported by 24 participants, 21 $(87.5 \%)$ of whom were not atopic.

\section{Conclusion}

The authors have identified a set of questions that correctly predict negative skin prick tests to common aeroallergens $88 \%$ of the time. These may be useful to exclude patients who do not warrant further investigation and who can reliably be advised that allergen avoidance is neither necessary nor helpful.

\section{Keywords}

allergy; atopy; general practice; IgE-mediated; primary care; skin prick test.

\section{INTRODUCTION}

Allergic (lgE-mediated) disorders as a whole are responsible for substantial morbidity, healthcare utilisation, and costs to the NHS. ${ }^{1,2}$ In addition, a significant number of people mistakenly believe they have allergies and use both NHS and their own time and resources pursuing unnecessary investigations (for example, conventional skin prick testing [SPT] and specific lgE blood testing, both of which are associated with a high level of false positives), ${ }^{3}$ and in pursuing alternative allergy testing (such as hair analysis or kinesiology, which have no scientific basis). As a result they may unnecessarily avoid exposure to presumed triggers.

However, it can often be difficult to differentiate between allergic (that is, IgE-mediated) and non-allergic (non-lgEmediated) symptoms. To date, the only way of identifying non-atopic status /defined as negative allergy tests to one or more airborne allergens and independent of clinical symptoms) is to carry out SPT or blood tests for the presence of specific lgE. However, these diagnostic tests are expensive (in the case of blood tests) and/ or are often unavailable in the primary care setting (SPT). ${ }^{5}$ Furthermore, it is difficult for generalist healthcare professionals and people buying over-the-counter bloodtesting kits to interpret the results because of the high rate of false positives.

\footnotetext{
VS Hammersley, PhD, research fellow; A Sheikh OBE, MD, MSc, FRCP, FRCGP, FRCPE, FRSE, FFPH, FACMI, FMedSci, professor of primary care research and development; E Davidson, $\mathrm{MBChB}$, MPH, FNZCPHM, research fellow, Usher Institute of Population Health Sciences and Informatics, University of Edinburgh, Edinburgh. J Harris, PhD, research fellow, Clinical Trials and Evaluation Unit, University of Bristol, Bristol. S Walker, PhD, deputy chief executive and director of research and policy, Asthma UK, London; honorary lecturer Usher Institute of Population Health Sciences and Informatics, University of Edinburgh, Edinburgh.
}

Food allergy is a less common manifestation of lgE-mediated disease. It is commonly overestimated, particularly among parents, who often attribute childhood skin rashes (which may be virally induced) to food allergy. IgE-mediated food allergy is uncommon in those who are non-atopic (that is, not sensitised to common airborne allergens as shown by negative skin prick/specific lgE tests to those allergens). In a 2005 study, less than $0.2 \%$ of children who were non-atopic went on to test positive to one of 12 food allergens. ${ }^{6}$ In adults, $10-12 \%$ of the general adult population think they have some type of food allergy or intolerance, ${ }^{7,8}$ even though IgE-mediated food allergy can only be confirmed in $1-2 \%{ }^{6}$ This represents a fivefold overestimation of food allergy, which is likely to have significant cost and societal implications. ${ }^{1,9}$

A screening tool that excludes allergy and allows the general public, nurses, or doctors to identify people who are nonatopic could bring significant resource and health benefits. A simple, inexpensive, non-invasive method of screening has the potential to reduce non-essential testing and referral land therefore healthcare use and costs to the NHS), and to reassure patients about the safety of continued exposure to allergens they wrongly suspect of causing their symptoms. Similar studies using a diagnostic tool to exclude

\section{Address for correspondence}

Victoria S Hammersley, Usher Institute of Population Health Sciences and Informatics, Nine Edinburgh Bioquarter, 9 Little France Road, Edinburgh, EH16 4UX, UK.

E-mail: vicky.hammersleyded.ac.uk

Submitted: 23 May 2016; Editor's response: 21 June 2016; final acceptance: 23 August 2016. CBritish Journal of General Practice This is the full-length article (published online 28 Feb 2017) of an abridged version published in print. Cite this version as: Br J Gen Pract 2017; DOI: https://doi.org/10.3399/bjgp17X689869 


\section{How this fits in}

The purpose of this study was to identify simple questions that can accurately predict the absence of atopy without the need for expensive and time-consuming objective tests, for example, skin prick tests or allergen-specific lgE blood tests. These questions can now be developed into a screening tool to be used by healthcare professionals and the general public to exclude allergy. This will prevent unnecessary avoidance of allergens, and could also be useful in excluding an allergic basis for adverse reactions to food, which is extremely uncommon in those who are non-atopic.

disease in other medical fields include the use of a D-dimer test in patients with suspected venous thromboembolism ${ }^{10,11}$ and the measurement of serum levels of procalcitonin as a marker for bloodstream infections. ${ }^{12}$

Data from a large paediatric cohort study $^{13}$ suggest that approximately five to 10 questions from a patient's clinical history may accurately predict non-atopic status, but this requires testing in clinical practice to confirm utility. The aim of this study was therefore to investigate whether key clinical questions could accurately identify patients who are non-atopic.

\section{METHOD}

\section{Participants}

Adults aged $>16$ years registered with general practices, or members of the general public, in Lothian, Scotland, were invited to participate in this study. It was performed in 2012. The exclusion criteria were:

- <16 years old;

- pregnancy;

- uncontrolled asthma;

- a previous history of anaphylaxis;

- antihistamines taken in the previous 48 hours; and

\section{Box 1. Ashford Birth Cohort Study screening questions}
1. Do you have a personal history of hayfever?
2. Do you have a personal history of asthma?
3. Do you have a personal history of eczema or asthma as a baby (age <2 years)?
4. Do you have a personal history of other allergy?
5. Do any of your parents or siblings have a history of hayfever?

- unwilling or unable to give informed consent.

\section{Recruitment}

The study was advertised in general practices, in liaison with the Scottish Primary Care Research Network. Posters were displayed in the waiting areas of the practices, and flyers were handed out to those who expressed an interest. Flyers were also sent to patients attending designated asthma clinics. E-mail, posters, and websites within the University of Edinburgh were used to inform the general public. Potential participants were asked to contact the researcher by phone or e-mail and were sent the participant information sheet to read before their appointment. Participants were asked to book an appointment with the researcher for clinics at their GP surgery or in the clinical research facility at the Royal Infirmary of Edinburgh. Consent forms were completed and exclusion criteria checked at the beginning of the appointment. Permission was obtained for the results of the skin prick testing to be sent to the participant's GP.

\section{Questionnaire development (index test)}

The authors identified five questions from the Ashford Birth Cohort Study' ${ }^{13}$ that had the potential to accurately predict nonatopic status, but which had not been tested in clinical practice, and used them as the basis for the questionnaire (Box 1). Cullinan and colleagues (2004) $)^{13}$ defined atopy as a positive skin prick test $(\geq 3 \mathrm{~mm})$ to at least one of mixed grasses, cat fur, and house dust mite. Parents in the Ashford Cohort answered the five allergy questions, and parents and children were skin prick tested. The number of people who were non-atopic in relation to each negative response ranged from 63 to $77 \%$; that is, if a parent reported that they had no history of hayfever then they had a negative allergy test to grass $77 \%$ of the time (high negative predictive value). The authors amended the wording of these questions and supplemented them with six additional questions, identified through consultation with experienced allergy clinicians, which also had the potential to discriminate between allergy and nonallergy. Five allergy clinicians were sent a short summary of the study prior to the consultation (Appendix 1) and asked to identify any additional questions that they felt were able to discriminate between atopy and non-atopy in a person presenting with suspected allergy. All suggestions were included, and the final 11 questions are shown in Box 2. 


\section{Box 2. Questions used in screening tool}

\section{Are you aged $<40$ years?}

2. Do you have, or have you ever had, hayfever?

3. Do any of your parents or siblings (brothers or sisters) have, or have they ever had, hayfever?

4. Do you have, or have you ever had, asthma?

5. Did you ever have eczema or asthma as a baby (aged <2 years)?

6. Do you have, or have you ever had, any other allergy?

7. Do you ever have any symptoms of itch or sneeze?

8. Do your allergy symptoms vary when you go from place to place (for example, on holiday)?

9. Is there a specific trigger that always sets off your allergy symptoms?

10. Do your allergy symptoms start within 30 minutes of being exposed to a specific trigger?

11. Do your allergy symptoms improve after treatment with antihistamines? predictive value (PPV) was the proportion of patients with a positive history who were atopic, and the negative predictive value (NPV) was the proportion of patients with a negative history who were not atopic.

Logistic regression techniques were used to identify the combination of questions that demonstrated the strongest association with non-atopic status. Responses to all questions were considered individually, then a multivariate model was constructed by including the questions with the strongest associations one by one until no other questions significantly contributed to the fit of the final model. The contribution of each question was evaluated using likelihood ratio tests. Once these questions were identified it was possible to calculate the sensitivity, specificity, and positive and negative predictive values for this combination of questions. Participants were defined as questionnaire positive if at least one response to these questions was positive, and questionnaire negative if all responses were negative. All statistical analyses were undertaken using Stata (version 13).

\section{Sample size}

Based on a sample size of 150 , with $95 \%$ Cls of $\pm 4.8 \%$, specificity would be estimated at $90 \%$. The sample size was calculated using an online statistical tool. ${ }^{14}$

\section{RESULTS}

In all, 143 participants completed the questionnaire and underwent skin prick tests. No adverse reactions occurred. There were no indeterminate index or reference standard results or missing data. The mean age of participants was 41.1 years (range $18.8-84.9)$ and $76.9 \%$ were female. A total of 81 participants $(56.6 \%)$ were atopic. The rates for each allergen were 62/143 (43.4\%) for grass, $67 / 143(46.9 \%)$ for house dust mite, 49/143 (34.3\%) for cat, and 33/143 (23.1\%) for dog. Individual question analysis is shown in Table 1.

The authors explored any correlation between questions and, although many of the questions were related to each other, none was in perfect agreement. Logistic regression techniques identified Questions $2,8,3$, and 9 as being independently associated with the risk factor of being nonatopic (Table 2).

A negative response to Question 8 was the most associated with being non-atopic (that is, negative skin tests). After adjusting for the other questions, patients were four times more likely to have negative test results if they answered ' $N$ o' to that 
Table 1. Validity of each question as a tool for identifying atopic status

\begin{tabular}{|c|c|c|c|c|c|}
\hline Question & $\begin{array}{l}\text { Negative response } \\
\text { to question, } n(\%)\end{array}$ & $\begin{array}{l}\text { Sensitivity, } \\
\%(95 \% \mathrm{Cl})\end{array}$ & $\begin{array}{l}\text { Specificity, } \\
\%(95 \% \mathrm{Cl})\end{array}$ & $\begin{array}{l}\text { Positive predictive } \\
\text { value, } \%(95 \% \mathrm{Cl})\end{array}$ & $\begin{array}{l}\text { Negative predictive } \\
\text { value, } \%(95 \% \mathrm{Cl})\end{array}$ \\
\hline 1. Are you aged $<40$ years? & $73(51.1)$ & 53 (42 to 64) & 56 (43 to 69) & 61 (49 to 73$)$ & 48 (36 to 60 ) \\
\hline 2. Do you have, or have you ever had, hayfever? & 73 (51.0) & 68 (57 to 78) & 75 (63 to 86$)$ & 79 (67 to 87$)$ & 64 (52 to 75$)$ \\
\hline $\begin{array}{l}\text { 3. Do any of your parents or siblings (brothers or } \\
\text { sisters) have, or have they ever had, hayfever? }\end{array}$ & 79 (55.2) & 59 (48 to 70$)$ & $74(62$ to 84$)$ & 75 (63 to 85$)$ & $58(47$ to 69$)$ \\
\hline 4. Do you have, or have you ever had, asthma? & $96(67.1)$ & 43 (32 to 54) & 80 (69 to 90) & $74(60$ to 86$)$ & 52 (42 to 62$)$ \\
\hline 6. Do you have, or have you ever had, any other allergy? & 56 (39.2) & 69 (58 to 79) & 50 (37 to 62) & 64 (53 to 74$)$ & 55 (41 to 69$)$ \\
\hline 7. Do you ever have any symptoms of itch or sneeze? & 25 (17.5) & 91 (83 to 96) & 29 (18 to 42) & 62 (53 to 71$)$ & 72 (51 to 88$)$ \\
\hline $\begin{array}{l}\text { 8. Do your allergy symptoms vary when you go from } \\
\text { place to place (for example, on holiday)? }\end{array}$ & $61(43.0)$ & 75 (64 to 84) & 66 (53 to 78) & 74 (63 to 83 ) & 67 (54 to 79 ) \\
\hline $\begin{array}{l}\text { 9. Is there a specific trigger that always sets off } \\
\text { your allergy symptoms? }\end{array}$ & 76 (53.2) & 62 (50 to 72) & 73 (60 to 83) & 74 (63 to 84$)$ & 59 (47 to 70 ) \\
\hline $\begin{array}{l}\text { 10. Do your allergy symptoms start within } 30 \text { minutes } \\
\text { of being exposed to a specific trigger? }\end{array}$ & 64 (44.8) & 69 (58 to 79) & 63 (50 to 75) & 71 (60 to 81 ) & 61 (48 to 73 ) \\
\hline $\begin{array}{l}\text { 11. Do your allergy symptoms improve after } \\
\text { treatment with antihistamines? }\end{array}$ & $58(40.6)$ & 72 (61 to 81 ) & 56 (43 to 69) & 68 (57 to 78 ) & 60 (47 to 73 ) \\
\hline
\end{tabular}

question. Table 3 shows the relationship between the responses to these four questions and atopic status.

Comparison of the questionnaires and skin tests gave a sensitivity of $96.3 \%$ (78/81, $95 \% \mathrm{Cl}=89.6$ to 99.2 ), specificity of $33.9 \%$ $(21 / 62,95 \% \mathrm{Cl}=22.3$ to 47.0$)$, PPV of $65.6 \%$

\section{Table 2. Results from logistic regression ${ }^{\mathrm{a}}$}

\begin{tabular}{|c|c|c|}
\hline Negative response to: & $\begin{array}{l}\text { Adjusted odds } \\
\text { ratiob }^{\mathrm{b}}(95 \% \mathrm{Cl})\end{array}$ & $P$-value \\
\hline 2. Do you have, or have you ever had, hayfever? & 2.44 (0.99 to 6.00$)$ & 0.050 \\
\hline $\begin{array}{l}\text { 8. Do your allergy symptoms vary when you go from } \\
\text { place to place (for example, on holiday)? }\end{array}$ & 4.00 (1.67 to 9.57) & 0.002 \\
\hline $\begin{array}{l}\text { 3. Do any of your parents or siblings (brothers or sisters) } \\
\text { have, or have they ever had, hayfever? }\end{array}$ & 3.19 (1.37 to 7.44$)$ & 0.010 \\
\hline $\begin{array}{l}\text { 9. Is there a specific trigger that always sets off your } \\
\text { allergy symptoms? }\end{array}$ & 3.09 (1.32 to 7.22 ) & 0.010 \\
\hline
\end{tabular}

Table 3. Responses to the four key questions and atopic status

\begin{tabular}{lcccc} 
& \multicolumn{2}{c}{ Skin prick test } & Total, $\boldsymbol{n}$ \\
\cline { 2 - 4 } & Atopic, $\boldsymbol{n}$ & Not atopic, $\boldsymbol{n}$ & 119 \\
\hline Questionnaire positive $^{\mathrm{a}}$ & 78 & 41 & 24 \\
\hline Questionnaire negative $^{\mathrm{b}}$ & 3 & 21 & 143 \\
\hline Total, $n$ & 81 & 62 & \\
\hline
\end{tabular}

${ }^{a}$ At least one positive response. ${ }^{b}$ All responses negative.
(78/119, $95 \% \mathrm{Cl}=56.3$ to 74.0$)$, and NPV of $87.5 \%(21 / 24,95 \% \mathrm{Cl}=67.6$ to 97.3$)$.

This shows that the combination of Questions 2, 8, 3, and 9 had a reasonably high NPV. Most (87.5\%) of those who gave negative responses to all the questions were non-atopic (had negative skin tests).

\section{DISCUSSION}

\section{Summary}

The authors were able to identify four questions that were reasonably predictive of non-atopic status in patients with suspected allergy. This has the potential to be useful in differentiating between IgE-mediated and non-lgE-mediated symptoms land so to drive treatment choices and avoidance advice) in primary care, where diagnostic tests are not routinely available.

\section{Strengths and limitations}

The authors were able to recruit members of general practices and the general public for this study, and almost achieved the target sample size of 150 . The screening tool is intended for use by healthcare professionals in primary care and the general population, which is where it was tested, ${ }^{15}$ and was compared with the best gold standard available. The authors' definition of atopy, a positive skin prick test to common (countryand climate-specific) aeroallergens, has been used in other studies, ${ }_{1}^{13}$ and the choice of skin prick testing as a gold standard is safe and feasible for aeroallergens. ${ }^{16}$ However, 


\section{Funding}

This study was funded by the Chief Scientist Office, grant reference number: CZG 2 576. Jessica Harris was supported by the National Institute for Health Research Bristol Cardiovascular Biomedical Research Unit.

\section{Ethical approval}

Ethical approval was obtained from the South East Scotland Research Ethics Committee 02 (SES REC 02), reference 12/SS/0094. Research and development management approval was obtained from NHS Lothian. Amendment approval to extend the recruitment to the general public was obtained from SES REC 02.

\section{Provenance}

Freely submitted; externally peer reviewed.

\section{Competing interests}

The authors have declared no competing interests.

\section{Acknowledgements}

The authors would like to thank the participants who generously contributed their time to undergo allergy testing. They would also like to thank the healthcare practitioners involved in conducting this study, particularly the clinical research facility nurses and general practice nurses. The authors are also grateful to the lay representatives for their support and advice.

\section{Discuss this article}

Contribute and read comments about this article: bjgp.org/letters this definition of atopy gave a prevalence of $57 \%$ in the sample population, which is high compared with other similar studies, presumably because there was a higher motivation to participate among people who are atopic. ${ }^{17}$ This may have resulted in loss of power to detect important questions that predict non-atopy.

A further limitation is the sample size. The numbers were small in the detailed analysis, where combinations of questions were examined, and increasing the sample population would have contributed more data to each combination of positive/ negative results to the skin prick testing and the questions, potentially increasing the negative predictive values and improving precision.

Due to the nature of the recruitment advertising with posters and leaflets - the authors were unable to record how many people saw the information but declined to take part, thus preventing any consideration of how representative the findings are.

PPV of $98.65 \%$, and NPV of $85.59 \%$. Similarly, in a study looking at the accuracy of allergy skin prick tests, ${ }_{17}^{17}$ SPT was predicted to be positive in $42.6 \%$ of cases and was actually positive in $36.1 \%$. In SPT results with a cutoff value of $3 \mathrm{~mm}$, prediction sensitivity was $77 \%$, specificity was $65.3 \%$, PPV was $65 \%$, and NPV was $86 \%$. In studies of different diagnostic tests, Wang and colleagues ${ }^{11}$ reported a slightly lower sensitivity of $64 \%$ and a higher NPV of $94 \%$, concluding that the D-dimer assay may have a role in tailoring treatment to optimise prevention of venous thromboembolism. Similar results were found for the predictive value of procalcitonin in excluding bloodstream infections and managing antibiotic usage (83\% sensitivity and $94 \%$ NPV). ${ }^{12}$

When a test has a high sensitivity, a negative test rules out the diagnosis, ${ }^{21}$ and this study reports a high sensitivity (96\%) and NPV (88\%). The authors are therefore fairly confident that this result using the four questions has a high NPV las it is almost 90\%), although the numbers were relatively small.

\section{Comparison with existing literature}

Previous work in this field has mainly focused on developing questions that can accurately predict sensitisation to a suspected allergen Ithat is, whether positive answers to clinical questions predict positive skin prick or specific IgE test results). ${ }^{17-19}$ The focus of this current study, however, was different. The authors sought to identify simple questions for which negative answers could predict negative skin tests in a general population, with a view to being able to assess atopic status without the need for a formal diagnostic test

This combination of questions compares well with other studies that have reported an NPV for atopy, including the ALATOP20 in vitro multispecific lgE test, which reported sensitivity of $89.57 \%$, specificity of $98.06 \%$,

\section{Implications for research and practice}

The authors were able to identify four questions that were predictive of non-atopic status. The results provide useful data for the development of a screening tool for non-atopic status in people with suspected allergy, although the questions need further validation in a larger, independent population of consecutively enrolled in the combination analysis, and increase precision.

The screening tool could then be confidently used by healthcare professionals or patients to accurately predict non-atopic status. patients. This would increase the numbers 


\section{REFERENCES}

1. Anandan C, Gupta R, Simpson C, et al. Epidemiology and disease burden from allergic disease in Scotland: analyses of national databases. J R Soc Med 2009 102(10): 431-442.

2. Gupta R, Sheikh A, Strachan DP, Anderson HR. Burden of allergic disease in the UK: secondary analyses of national databases. Clin Exp Allergy 2004; 34(4): 520-526.

3. Soares-Weiser K, Takwoingi Y, Panesar SS, et al. The diagnosis of food allergy: a systematic review and meta-analysis. Allergy 2014; 69(1): 76-86.

4. Burton C, Irshad T, Sheikh A. Understanding the experiences of allergy testing a qualitative study of people with perceived serious allergic disorders. Postgrad Med J 2010; 86(1020): 591-596.

5. Sheikh A, Levy ML. Costs are a barrier to GPs performing skin prick testing. $\mathrm{Br}$ J Gen Pract 1999; 49(438): 67

6. Roberts G. Peckitt C, Northstone K, et al. Relationship between aeroallergen and food allergen sensitization in childhood. Clin Exp Allergy 2005; 35(7): 933-940.

7. Vierk KA, Koehler KM, Fein SB, Street DA. Prevalence of self-reported food allergy in American adults and use of food labels. J Allergy Clin Immunol 2007; 119(6): 1504-1510.

8. Woods RK, Abramson M, Bailey M, Walters EH. International prevalences of reported food allergies and intolerances. Comparisons arising from the European Community Respiratory Health Survey (ECRHS) 1991-1994. Eur J Clin Nutr 2001; 55(4): 298-304.

9. National Institute for Health and Clinical Excellence. Food allergy in children and young people. Diagnosis and assessment of food allergy in children and young people in primary care and community settings. CG116. London: NICE, 2011. https://www.nice.org.uk/guidance/cg116/evidence/fullguideline-136470061 (accessed 20 Feb 2017).

10. Di Nisio M, Squizzato A, Rutjes AW, et al. Diagnostic accuracy of D-dimer test for exclusion of venous thromboembolism: a systematic review. J Thromb Haemost 2007; 5(2): 296-304

11. Wang $Y$, Liu ZH, Zhang HL, et al. Predictive value of D-dimer test for recurrent venous thromboembolism at hospital discharge in patients with acute pulmonary embolism. J Thromb Thrombolysis 2011; 32(4): 410-416.

12. Wang $H$, Yin F, Shen DX, et al. Predictive value of procalcitonin for excluding bloodstream infection: results of a retrospective study and utility of a rapid, quantitative test for procalcitonin. J Int Med Res 2013; 41(5): 1671-1681.

13. Cullinan P, MacNeill SJ, Harris JM, et al. Early allergen exposure, skin prick responses, and atopic wheeze at age 5 in English children: a cohort study. Thorax 2004; 59(10): 855-861.

14. Lenth RV. Java applets for power and sample size. 2006-2009. http://www.stat uiowa.edu/ rlenth/Power laccessed 20 Feb 2017).

15. Galant SP, Crawford LJ, Morphew T, et al. Predictive value of a cross-cultural asthma case-detection tool in an elementary school population. Pediatrics 2004; 114(3): e307-e316.

16. Reid MJ, Lockey RF, Turkeltaub PC, Platts-Mills TA. Survey of fatalities from skin testing and immunotherapy 1985-1989. J Allergy Clin Immunol 1993; 92(1 Pt 1): 6-15

17. Karakaya G, Ozturk AB, Kalyoncu AF. Prediction of atopy by skin prick tests in patients with asthma and/or persistent rhinitis. Allergol Immunopathol (Madr) 2012; 40(1): 37-40

18. Lakwijk N, Van Strien RT, Doekes G, et al. Validation of a screening questionnaire for atopy with serum lgE tests in a population of pregnant Dutch women. Clin Exp Allergy 1998; 28(4): 454-458.

19. Smith HE, Hogger $C$, Lallemant $C$, et al. Is structured allergy history sufficient when assessing patients with asthma and rhinitis in general practice? J Allergy Clin Immunol 2009; 123(3): 646-650.

20. Nogueira JM, de Almeida MM, Fernandes JG, et al. ALATOP: sensitivity, specificity and predictive value of a new 'in vitro' screening test of atopy. Allerg Immunol (Paris) 1994; 26(3): 99-101.

21. Centre for Evidence-Based Medicine. SpPin and SnNout. 2017. http://wnw. http://www.cebm.net/sppin-and-snnout/ (accessed 20 Feb 2017).

22. Pastorello EA, Incorvaia $\mathrm{C}$, Ortolani $\mathrm{C}$, et al. Studies on the relationship between the level of specific lgE antibodies and the clinical expression of allergy: I. Definition of levels distinguishing patients with symptomatic from patients with asymptomatic allergy to common aeroallergens. J Allergy Clin Immunol 1995; 96(5 Pt 1): 580-587. 


\section{Appendix 1. Study summary for allergy specialists}

\section{Developing and testing a screening tool to accurately predict non-atopic status in patients with suspected allergy}

\section{Background}

Disorders such as asthma, rhinitis, and urticaria are extremely common in Scotland, potentially affecting up to one in three of the population. ${ }^{1}$ The commonest manifestations are respiratory and dermatological, and are often caused by exposure to allergens such as pollens and house dust mites, mediated through the production of allergen-specific lgE antibodies and subsequent histamine release. Allergic (lgE-mediated) disorders as a whole are responsible for substantial morbidity, healthcare utilisation ( $>4 \%$ of GP consultations and 1.5\% of hospital admissions in Scotland are for allergic diseases) and costs to the NHS. In addition, there are significant numbers of people who mistakenly believe they are allergic and who utilise both NHS and their own time and resources pursuing unnecessary investigations (for example, conventional skin prick testing (SPT) and specific lgE blood testing, which are both associated with a high level of false positives) and pursuing alternative allergy testing (for example, hair analysis or kinesiology, which have no scientific basis), as a result of which they may unnecessarily avoid exposure to presumed triggers. ${ }^{4}$ For example, $10-12 \%$ of the general adult population think they have some type of food allergy or intolerance, ${ }^{7.8}$ even though IgE-mediated food allergy can only be identified in 1-2\%. ${ }^{6}$ This represents a fivefold overestimation of food allergy, which is likely to have significant cost and societal implications. ${ }^{1.9}$

However, it can often be difficult to differentiate between allergic (that is, IgE-mediated) and non-allergic (that is, non IgE-mediated) symptoms. To date, the only way of identifying non-atopic status (defined as negative allergy tests to one or more airborne allergens and independent of clinical symptoms) is to carry out SPT or blood tests for the presence of specific lgE. These diagnostic tests are, however, expensive and often unavailable in the primary care setting, and are furthermore difficult to interpret by healthcare professionals and also people buying blood-testing kits over-the-counter. Negative allergy tests alone are a relatively accurate predictor of nonallergy, ${ }^{22}$ although it is not clear at present whether a negative allergy history accurately predicts negative allergy tests.

\section{Outline of this project}

This study will investigate whether key clinical questions can accurately identify patients who are non-atopic. We propose to develop an instrument that is comprised of validated questions, which can identify those patients for whom an allergy test is so likely to be negative that it is not worth doing (that is, a very high negative predictive value). This has the potential to be useful both for clinicians and patients in streamlining care in a cost-effective manner.

Collaboration with colleagues from Imperial College London has enabled us to analyse unpublished data from the Ashford Birth Cohort, ${ }^{13}$ providing useful information about parental and child allergy status. Parents answered the following five allergy questions and parents and children were skin prick tested:

1. Do you have a personal history of hayfever?

2. Do you have a personal history of asthma?

3. Do you have a personal history of eczema or asthma as a baby lage $<2$ years)?

4. Do you have a personal history of other allergy?

5. Do any of your parents or siblings have a history of hayfever?

Atopy was defined as a positive SPT ( $\geq 3 \mathrm{~mm}$ ) to at least one of mixed grasses, cat fur, and house dust mite. The numbers of people being non-atopic in relation to each negative response ranged from 63 to $77 \%$; that is, if a parent said that they had no history of hayfever then they had a negative allergy test to grass $77 \%$ of the time (high negative predictive value). A negative response to all the questions was associated with a high rate of negative allergy tests in adults (83\%). Results from the same study in children were similar (89\%), although numbers were smaller. High specificity (ideally $95 \%$ ) is required for the tool to be useful in clinical practice.

These five questions will be used as the basis for the screening questionnaire. Additionally, we will identify any other questions that, according to experienced allergy clinicians, discriminate between allergy and non-allergy, and test them with the questions identified above to create the combination with the highest negative predictive value.

We now wish to find out which questions, in addition to those listed above, discriminate between atopy and non-atopy in someone presenting with suspected allergy. Name:

Position:

Questions:

1.

2.

3. 